\title{
The Application of Computer Science and Technology in Engineering Construction Project
}

\author{
Wang Yao-sheng \\ Nanchang University of Vocations
}

\begin{abstract}
The development of science and technology promotes the development and updating of computer science and technology, which meanwhile also expands its application range. What's more, with the continuous development of computer science and technology, its application in terms of engineering construction project is becoming wider and wider, bringing along with a leap of reform. Besides, engineering construction project management is complicated and cumbersome and previous manual management costs a lot of time and energy, while the application of computer science and technology greatly improves its scientificity and modernization. This paper simply elaborates its situation as well as necessity, and focuses on the exploration of concrete application of computer science and technology in engineering construction project.
\end{abstract}

KeyWords-Computer Science and Technology; Engineering Construction Project; Application

\section{I.INTRODUCTION}

The contribution of computer science and technology to social development demonstrates in various fields, which greatly improves people's quality of life and working efficiency, facilitating the development of modern society. The continuous innovation and development of science and technology provide assistance for production, life, culture entertainment etc. Besides, the popular application of computer science and technology in social development effectively upgrade the civilization level of modern society. In the advanced science and technology development context, computer science and technology continue to improve and progress. What's more, computer continually develops toward the trend of human-orientation which greatly enriches people's life. At the same time, its development features are quick update with small size.

All along, the management of engineering construction project adopts traditional approaches, which are no longer applicable in modern time. In addition, personnel responsible for the project use unenlightened management approaches, who do not only have a good knowledge of management but also technology, which shall lead to chaotic situation. The adoption of computer science and technology management connects various departments and change information in daily work so that employees can carry out communication via internet which can realize the integration of technology and economy.

This paper analyzes the meaning of applying computer science and technology in engineering construction project and meanwhile discusses its application in engineering construction project. The function of computer science .

\section{II.TECHNOLOGY DEVELOPMENT}

The development of everything has double faces, when computer brings along with various benefits it also increases hidden safety problems. For example, sensational millennium bug, which has brought great threats on people's production and life. A glittering array of industries severely suffers from millennium bug which makes people realize the existing problems of computer technology. Therefore, how to make computer develop in a healthy and stable situation, reduce the threat on social development to maximize the service function of computer technology has become a hot topic for computer science and technology development.

The application quality of computer science and technology in the engineering construction project management in China has been greatly improved, yet inevitably have shortcomings, if which do not be effectively dealt with, it shall hinder the long-term development of China's construction enterprises. Judging from the present socio-economic development, it is positive in a relatively stable situation. Under such circumstances, China's construction industry presents a lively scene, which has become a key driving force for China's economic development. However, the market 
competition is fierce now and the construction industry also faces great challenges. In order to shine out in the harsh environment, one must improve the level of construction management and apply computer science and technology to engineering project management so as to realize the modernization of project management

\section{THE CORE FUNCTION OF COMPUTER TECHNOLOGY}

Computer has been universally applied and promoted throughout the nation after being developed for half a century. Judging from the earliest computer technology
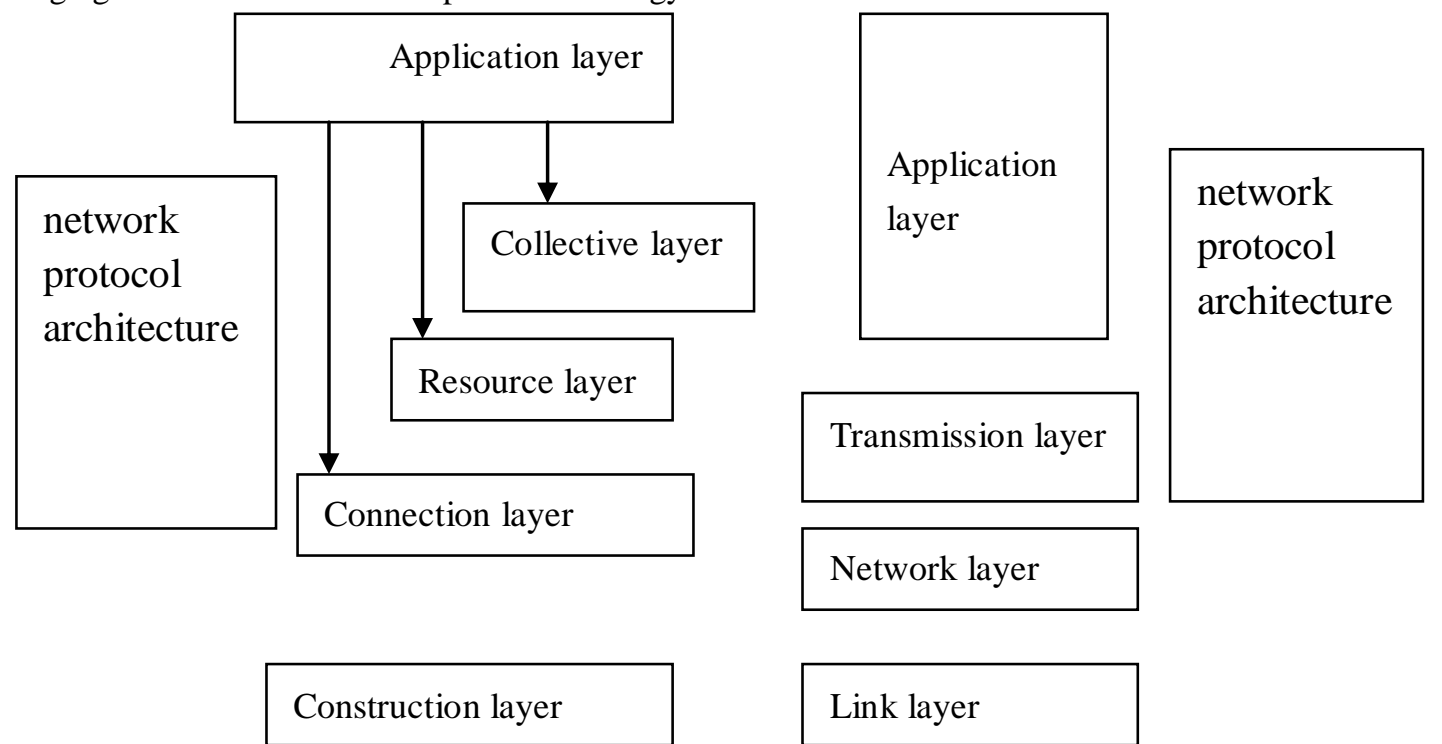

development, we can see that early computer technology simply count statistics while modern computer technology develops into network technology with various advantages of practicability, convenience, speed etc which effectively promotes the development of whole society. At present, the core function of computer technology mainly demonstrates in data processing and network application, showing in Fig .1 the computer network protocol architecture.

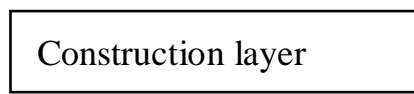

Figure 1. The computer network protocol architecture

Computer has powerful data processing function which is the operative technique frequently adopted. Data processing can effectively collect, compute and process data. Besides, it can automatically select data based on customer demand to realize high quality work. People only have to input the data to the computer memory and confirm executive order in the premise of preparing relevant program code to carry out comprehensive and automatic data processing which can guarantee working efficiency and quality. The automatic function of data processing is not applicable to employees of enterprise but also private individuals which is convenient, quick, simple and practical.

\section{IV.CONCRETE APPLICATION OF COMPUTER SCIENCE AND} TECHNOLOGY IN ENGINEERING CONSTRUCTION PROJECT MANAGEMENT

\section{A. Computer-assisted bidding evaluation}

While carrying out bidding, cost consulting, pre-assessment and rational decisions are very important preparatory programs, and computer-assisted bidding evaluation can realize scientific, reasonable and effective e-bidding evaluation. During the whole process of tendering and bidding, tenderees have to make electronic bidding files and send out with portable electronic storage devices. Then bidders fill out the file and count the cost to create reasonable and complete bidding documents and electronic bidding system.

\section{B. Control project cost based on computer}

In the course of engineering, project budget is a very important task which yet is difficult because the 
calculation is complicated which wastes lots of time and energy. In order to improve the general budget efficiency, relevant design and management sectors have widely applied computer program into general budget and assessment. Besides relevant software can print general budget documents based on concrete requirement which is quick with high efficiency, saving a lot of time, and avoiding complicated procedures, therefore, employees dealing with general budget and assessment can focus on economic analysis.

\section{CONCLUSION}

In the course of construction industry development in China, construction project management is always ignored yet the management level is closely related to the construction quality. Therefore, in order to improve the quality, we have to well implement the management and apply computer science and technology into management to facilitate the rapid development of China's construction industry.

This paper combines computer science as well as technology and engineering construction projects, and introduces computer service objects. The development of computer aims to better serve the society and applying computer science as well as technology into engineering construction projects is an inevitable trend which shall greatly push the continuous development of construction field.

\section{REFERENCES}

[1] Chen X. Department of Computer Science and Technology[J]. Journal of Henan Mechanical \& Electrical Engineering College, 2006

[2] Kent, A., Williams, J. G., Kent, R., \& Hall, C. M. (1975). Encyclopedia of computer science and technology. M. Dekker.

[3] Henderson, Harry. "Encyclopedia of Computer Science and Technology." Radiophysics \& Quantum Electronics 15.8(2008):38-39.

[4] Lichtenstein, Art A. "Encyclopedia of Computer Science and Technology." Booklist 26.8(2009):67-68.

[5] Dixit, R. K. "Global Journal of Computer Science and Technology. Volume 9, Issue 5 (Ver. 2.0).." Online Submission 9(2010): 160.

[6] Wang, A. M., Jie, L. I., Wang, D. L., Na, L. I., Wen-Ying, G. E., \& Yan-Qiang, G. E. (2010). National level characteristic major construction of computer science and technology. Computer Education, 2, 440 - 443.

[7] Liu C X, Guo M, Lan-Ying W U, et al. THE REFLECTION AND EXPERIENCE OF CHARACTERISTIC SPECIALTY OF COMPUTER SCIENCE AND TECHNOLOGY IN LOCAL UNIVERSITIES[J]. Journal of Jinggangshan University, 2011.

[8] Belzer, Jack. "Encyclopedia of Computer Science and Technology." Reference Reviews 26.8(1979):67-68.

[9] Chen J. Some Considerations for "Discrete Mathematics" Teaching in the Subject of Computer Science and Technology[J] Computer \& Telecommunication, 2011.

[10] Margenstern M. A Combinatorial Approach to HyperbolicGeometry as a New Perspective for Computer Science and Technology.[C]// Computers and Their Applications. 2003:468-471.

[11] Jiang Y. Problems and Countermeasures of Graduation Design in Computer Science and Technology at Local University[J]. Journal of Qujing Normal University, 2013.

[12] Hui Y U, Qing-Wei X U, Yang L. Research and Implementation on University-Enterprise Cooperation Graduation Design Model of Computer Science and Technology[J]. Journal of Hubei University of Education, 2013. 